\title{
ESTUDO DA PREVALÊNCIA DE LESÕES EM MUCOSA ORAL DE PACIENTES PORTADORES DE NECESSIDADES ESPECIAIS
}

\author{
Alena Peixoto Medrado*, Daniela A. R. Chaovi Silva**, Flávia Godinho C. Wanderley** \\ Autor correspondente: Alena Peixoto Medrado alenamedrado@hotmail.com \\ * Professora da Escola Bahiana de Medicina e Saúde Pública, Brasil. Doutora em Patologia Humana \\ ** Aluna de Iniciação Científica do Curso de Odontologia da Escola Bahiana de Medicina e Saúde Pública
}

\section{Resumo}

A odontologia especial tem como diretriz a prevenção, o diagnóstico e o tratamento de lesões bucais que acometem o sistema estomatognático. Em particular, os pacientes portadores de necessidades especiais representam uma população que apresenta desafios a serem transpostos para o sucesso do atendimento odontológico. O objetivo deste estudo qualiquantitativo foi avaliar as lesões bucais mais frequentes em pacientes especiais usuários de dois serviços de saúde. Ao longo de um ano, foram analisados os prontuários de 60 pacientes e dados relativos à idade, sexo, queixa principal, presença ou não de alterações bucais foram registrados. Em seguida, foi realizado exame bucal para verificação de possíveis patologias presentes na mucosa oral desta população. A amostra foi constituída por um maior número de indivíduos do sexo masculino $(53,3 \%)$ e por aqueles que se autodeclararam como negros (35\%). A lesão mais frequente foi a Hiperplasia fibrosa inflamatória (40\%), seguida por $\mathrm{Hi}$ perplasia gengival $(26,6 \%)$ e Candidíase $(11,6 \%) .18,3 \%$ dos indivíduos relataram xerostomia. Entre as drogas mais utilizadas pelos pacientes, destacaram-se os anti-hipertensivos (18,3\%), anti-convulsivantes $(8,3 \%)$ e anti-diabetogênicos $(8,3 \%)$. Muitos destes pacientes e ou responsáveis queixaram-se da dificuldade de acesso ao atendimento odontológico. Sendo assim, faz-se necessário um maior grau de atenção dedicado a esta população a fim de que o tratamento odontológico torne-se mais acessível.

Palavras-chave: Medicina bucal; Sistema Estomatognático; Unidade Hospitalar de Odontologia. 


\title{
PREVALENCE STUDY OF ORAL SOFT TISSUE LESIONS OF PATIENTS WITH SPECIAL NEEDS
}

\begin{abstract}
AIM: The aim of this study was to evaluate qualiquantitative the most frequent oral lesions in special patients using two health centers. METHODS: Over a year, the records of 60 patients were assessed and data on age, gender, main complain, presence or absence of oral abnormalities, recorded were analyzed. Then an oral exam was performed to check possible pathologies in the oral mucosa of this population. RESULTS: The sample consisted of a greater number of males (53.3\%) and those who declared themselves as black (35\%). The most common lesion was inflammatory fibrous hyperplasia (40\%), followed by gingival hyperplasia $(26.6 \%)$ and candidiasis (11.6\%). $18.3 \%$ of subjects reported perception of xerostomia. Many of these patients and or caregivers complained of difficulty in accessing dental care. CONCLUSION: Therefore, it is necessary a greater attention devoted to this population for dental care becomes more humanized.
\end{abstract}

Kepwords: Stomatognathic system.

\section{INTRODUÇÃO}

A odontologia especial tem como diretriz, o diagnóstico, prevenção, tratamento e controle das afecções do sistema estomatognático em pacientes portadores de necessidades especiais. ${ }^{(1)}$ Fazem parte dessa classificação pacientes portadores de doenças cardiovasculares, pulmonares, do apareIho geniturinário, e desordens de natureza endócrina, metabólica, imunológica, hematológica e oncológica. Procedem ainda, neste campo de atuação, os pacientes que apresentam distúrbios Psiquiátricos, neurológicos e comportamentais. ${ }^{(2)}$

Estudos descritos na literatura demonstram que as terapias medicamentosas realizadas com fármacos destinados ao tratamento de pacientes especiais propiciam alterações na cavidade oral. O sistema nervoso autônomo é o principal responsável pelo controle da secreção salivar, entretanto diversos hormônios podem alterar a sua composição. (3) Por exemplo, tem sido relatado que algumas drogas podem promover diminuição da atividade salivar e mudanças na microbiota oral.(4) Outros estudos tem demonstrado que os pacientes especiais apresentam com frequência diversas patologias bucais, a exemplo de candidíase, estomatites, ulcerações e síndrome da ardência bucal, entre outras. ${ }^{(5)}$

Tendo em vista o significativo número de lesões bucais que estes pacientes apresentam, em especial aquelas influenciadas pelas medicações normalmente utilizadas por esta população, a realidade do atendimento aos pacientes especiais revela que este grupo de indivíduos ainda enfrenta grandes dificuldades de acesso ao tratamento odontológico. Estas podem ser representadas por fatores diversos, tais como a região onde habitam, demanda de vagas disponíveis, a especialidade dos profissionais que atuam no serviço, indicação de outro profissional, acesso ao local do atendimento, acessibilidade ao transporte, custo do tratamento e aspectos sócio-culturais do paciente e de sua família. ${ }^{(6)}$

Muitas vezes as doenças sistêmicas apresentadas, despertam sentimentos de receio e medo por 
parte dos profissionais de saúde que se deparam com tais pacientes. Aliado a tal fato, verificou-se a necessidade de um maior tempo gasto no atendimento desta população. Por falta de preparação destes profissionais alguns aspectos da saúde oral destes indivíduos acabam por ser negligenciados. (7) É competência do cirurgião dentista se manter informado sobre as condições sistêmicas dos seus pacientes e preparado para adequá-los a um protocolo de atendimento odontológico especializado, o qual normalmente requer uma atenção mais personalizada em virtude dos possíveis fatores complicadores da saúde bucal decorrentes de tratamentos sistêmicos.

Em virtude do crescimento da população com necessidades especiais nas últimas décadas, gerado pelo aumento da expectativa de vida destes indivíduos, ${ }^{(8)}$ urge a necessidade de se desenvolver estudos voltados para a atenção às lesões bucais que podem eventualmente se manifestar nesta população.

A presente pesquisa objetiva avaliar as lesões bucais que podem ser detectadas em pacientes portadores de necessidades especiais. Pretende-se também traçar um perfil desta população-alvo no tocante à utilização dos fármacos. Desse modo, espera-se contribuir para uma melhor compreensão das condições de saúde bucal desta população e identificar as possíveis lesões em mucosa oral decorrentes do uso de fármacos por parte destes pacientes.

\section{MATERIAL E MÉTODOS}

A pesquisa foi desenvolvida nas dependências da Escola Bahiana de Medicina e Saúde Pública e Hospital Geral Roberto Santos, após passar pela aprovação do Comitê de Ética em Humanos da Escola Bahiana de Medicina e Saúde Pública e ser registrada pelo número 410.468 .

Foi realizado um estudo observacional qualiquantitativo de corte transversal que abrangeu o período de 12 meses (2013/2014), cuja amostra foi composta por 60 pacientes, dos quais 40 foram provenientes da Clínica de Odontologia Especial do Ambulatório Assistencial da Escola Bahiana de Medicina e Saúde Pública e 20 do Ambulatório para pacientes especiais do Hospital Geral Roberto Santos. Aos pacientes e/ou seus responsáveis foi apresentado o Termo de Consentimento Livre e Esclarecido, para a decisão de se tornarem voluntários ou não da pesquisa. Os prontuários destes pacientes foram analisados e dados relativos ao sexo, idade, cor da pele, queixa principal, condição sistêmica, presença ou não de lesão bucal, medicação em uso e percepção de xerostomia ou sialorreia foram coletados e categorizados. As informações registradas nos prontuários com registro de informações de menores e/ou pacientes portadores de alterações cognitivas foram relatadas por seus respectivos responsáveis legais. Os pacientes foram classificados em grupos definidos pela condição sistêmica que apresentavam, a saber Diabetes Mellitus, Hipertensão Arterial, Doenças neuropsicomotoras (decorrentes de paralisia cerebral e/ou outros comprometimentos do sistema psicomotor), Síndromes Genéticas (Down, Sturge Weber e Wilson; entre outras), Insuficiência Renal Crônica, Hepatites e Cirrose.

Um único examinador calibrado realizou o exame bucal para a verificação de possíveis patologias presentes em mucosa, utilizando espátula de madeira e gaze estéril. Durante a realização do exame foram pesquisadas lesões bucais que se desenvolvem em mucosa tais como Hiperplasia fibrosa inflamatória, hiperplasia gengival, hipercetatose, candidíase e lesões aftosas. Caso fossem constatados outros tipos de lesões, estes dados também eram computados na planilha em EXCEL construída especificamente para o estudo.

Para a variável quantitativa (idade), obtiveram-se valores correspondentes à média aritmética. No que diz respeito às variáveis qualitativas (gênero, procedência, história médica e odontológica e tipo de necessidade especial), os dados foram obtidos a partir de uma tabela unidimensional de frequência, a partir da qual foram identificados os seus respectivos percentuais. 


\section{RESULTADOS}

A amostra compreendeu 60 pacientes, sendo 40 indivíduos provenientes do ambulatório da Clinica de Odontologia Especial e 20 do ambulatório para Pacientes Especiais do Hospital Geral Roberto Santos. A idade dos pacientes variou entre 4 a 81 anos, com média de 39,35. Dos participantes da pesquisa examinados, $32(53,3 \%)$ pertenciam ao gênero masculino e 28 (46,6\%) ao feminino. Informações referentes à percepção da etnia dos indivíduos revelaram que $21(35 \%)$ se autoconsideraram melanodermas, ao passo que $19(31,6 \%)$ e $16(26,6 \%)$, se descreveram como leucodermas e faiodermas, respectivamente (Tabela 1 ).

Tabela 1 - Características da amostra relacionadas ao gênero e autopercepção de cor da pele

\begin{tabular}{lcc}
\hline \multicolumn{1}{c}{ VARIÁVEIS } & N & $\%$ \\
\hline Gênero & 28 & 46,6 \\
Feminino & 32 & 53,3 Cor da Pele \\
Masculino & 21 & 35 \\
Melanoderma & 19 & 31,6 \\
Leucoderma & 16 & 26,6 \\
Faioderma & & \\
\hline
\end{tabular}

A queixa principal da procura ao Serviço foi tratamento e/ou limpeza para cerca de $30 \%$ dos examinados, exodontia $(21,6 \%)$ e $10 \%$ relataram que precisavam de adequação bucal para transplante renal ou hepático.
As condições sistêmicas mais prevalentes na amostra foram as desordens neuropsicomotoras $(41,6 \%)$, seguidas por síndromes genéticas $(26,6 \%)$ e hipertensão arterial $(21,6 \%)$ (Tabela 2$)$.

Tabela 2 - Distribuição das doenças sistêmicas registradas nos prontuários dos pacientes

\begin{tabular}{lcc}
\hline \multicolumn{1}{c}{ CondiçÃo SISTÊMICA } & N & $\%$ \\
\hline Doenças Neuropsicomotoras & 25 & 41,6 \\
Síndromes Genéticas & 16 & 26,6 \\
Hipertensão Arterial & 13 & 21,6 \\
Diabetes Mellitus & 7 & 11,6 \\
Doenças Hepáticas & 5 & 8,3 \\
Insuficiência Renal Crônica & 4 & 6,6 \\
Outros & 8 & 13,3 \\
\hline
\end{tabular}

Quando inqueridos acerca de possíveis alterações no fluxo salivar decorrentes do uso contínuo de medicações, 24 pacientes (40\%) relataram uma condição de normalidade do fluxo salivar. $18,3 \%$ dos pacientes da amostra descreveram a sensação de xerostomia e 10\% queixaram-se de sialorréia (Tabela 3). 
Tabela 3 - Avaliação da autopercepção do fluxo salivar dos participantes do estudo

\begin{tabular}{lcc}
\hline \multicolumn{1}{c}{ Fluxo SalivaR } & N & $\%$ \\
\hline Xerostomia & 11 & 18,3 \\
Normal & 24 & 40 \\
Sialórreia & 6 & 10 \\
\hline
\end{tabular}

Ao se avaliar as classes de fármacos utilizados pelos indivíduos do estudo, observou-se que os mais usados foram os anti-hipertensivos (18,3\%), anticonvulsivantes e antidiabetogênicos $(8,3 \%)$, e antipsicóticos e ansiolíticos (6,6\%) (Tabela 4). $21,6 \%$ de todos os pacientes examinados relataram não fazer uso de nenhum tipo de medicamento.

Tabela 4 - Categorias farmacológicas utilizadas pelos pacientes

\begin{tabular}{lcc}
\hline \multicolumn{1}{c}{ CATEgORIA FARMACOLÓGICA } & N & $\%$ \\
\hline Anti-hipertensivos & 11 & 18,3 \\
Anticonvulsivantes & 5 & 8,3 \\
Antidiabetogênicos & 5 & 8,3 \\
Antipsicóticos & 4 & 6,6 \\
Ansiolíticos & 4 & 6,6 \\
Não fazem uso de medicação & 13 & 21,6 \\
\hline
\end{tabular}

O exame intrabucal possibilitou diagnosticar uma variedade de lesões orais em mucosa. Dentre estas, a Hiperplasia Fibrosa Inflamatória foi a condição mais frequente e representou $40 \%$ da amos- tra, seguida pela Hiperplasia Gengival (26,6\%) e Candidíase (11,6); (Tabela 5). Vinte pacientes (33,3\%) não exibiram nenhum tipo de lesão em mucosa, independente da sua localização.

Tabela 5 - Lesões mucosas orais visualizadas durante a realização do exame clínico intrabucal

\begin{tabular}{lcc}
\hline \multicolumn{1}{c}{ LESÕES } & N & $\%$ \\
\hline Hiperplasia Fibrosa Inflamatória & 24 & 40 \\
Hiperplasia Gengival & 16 & 26,6 \\
Candidíase & 7 & 11,6 \\
Afta Recorrente & 3 & 5 \\
Abcessos & 3 & 5 \\
Outras Alterações & 4 & 6,6 \\
Não apresentaram alteração & 20 & 33,3 \\
\hline
\end{tabular}




\section{DISCUSSÃO}

Esta pesquisa teve como objetivo principal avaliar a ocorrência de lesões orais na mucosa de pacientes portadores de necessidades especiais. Esta população-alvo foi escolhida em virtude da crescente demanda existente para esta especialidade e a carência de Serviços de Saúde voltados para o atendimento a estes indivíduos. No presente estudo, muitos destes pacientes e ou responsáveis queixaram-se da dificuldade de acesso ao atendimento odontológico, alegando a existência de poucos locais com estrutura mínima necessária para atendimento. Relataram ainda a falta de capacitação por parte de alguns cirurgiões dentistas e a necessidade de atenção multidisciplinar nos postos de saúde das suas regiões. Estes achados têm sido relatados por alguns autores que analisaram especificamente a acessibilidade e possíveis fatores complicadores ao atendimento dos pacientes especiais nos Serviços de Saúde existentes. ${ }^{(6)}$ Em nosso estudo, muitos indivíduos eram provenientes do interior do Estado da Bahia e contavam apenas com o apoio da Prefeitura local para o seu deslocamento, o qual geralmente era realizado em meios de transporte inapropriados que representavam uma condição de estresse para os pacientes. Segundo Katz $(2012)^{(9)}$ para 0 atendimento a pacientes especiais é necessário um ambiente tranquilo, desprovido de condições estressantes para que se consiga atingir os objetivos delineados para o tratamento odontológico de qualidade e possa se atingir um nível de excelência no atendimento que seja facilmente percebido pelos usuários do serviço.

$\mathrm{Na}$ presente investigação, observou-se que os indivíduos queixaram-se primariamente da necessidade de realizar "limpeza" e "extrair dente", fato este que corrobora a realidade do tratamento odontológico descrita na literatura e reflete a necessidade do desenvolvimento de uma abordagem preventiva para esta população. Muitas vezes, a percepção da condição de saúde bucal por parte deste paciente está condicionada à sua condição sistêmica. Por exemplo, tem sido relatado que pacientes portadores de distúrbios neuropsicomoto- res apresentam maior grau de dificuldade para higienizar a cavidade oral em virtude do déficit da função do aparelho locomotor. ${ }^{(10)}$

O fluxo salivar dos pacientes estudados foi avaliado através da autopercepção deste, após o início do uso da medicação para o tratamento da sua condição sistêmica. Amaral (2009) ${ }^{(5)}$ realizou uma revisão de grupos farmacológicos causadores de alterações específicas na cavidade oral. Os autores relataram que os fármacos utilizados por estes pacientes estão relacionados a quadros de xerostomia e alteração da função e composição salivar. Foram citadas drogas antieméticas como a promatazina; anti-hipertensivos como atenolol, captopril, clomidina, metropolol; ansiolíticos a exemplo do clonazepam, lorazepam, diazepam e clonazepam; antidiabetogênicos tais como a hidroclorotiazida e furosemida; anticonvulsivantes, antipsicóticos e antiulcerosos (carbomazepina, respiridona e omeprazol, respectivamente). Foi relatado o uso de muitas destas classes de fármacos por parte da amostra do presente estudo. As drogas mais utilizadas foram captopril, carbamazepina, diazepam, hidroclorotiazida e omeprazol, algumas das quais podem induzir quadros de hipossalivação. Alguns fármacos relatados propiciaram também o desenvolvimento de hiperssalivação, entre os quais destacaram-se o lorazepam e a respiridona. Tendo em vista a diversidade de medicamentos utilizadas pela população do estudo, urge a necessidade de investigações adicionais que estabeleçam uma relação de causa e efeito entre as drogas citadas e suas respectivas repercussões na cavidade oral dos seus usuários, uma vez que tal associação não foi contemplada no presente estudo.

A despeito da condição de saúde gengival destes indivíduos, que muitas vezes apresentava-se deficiente, a avaliação periodontal não integrou o escopo da presente investigação. A lesão em mucosa oral mais frequente foi a hiperplasia fibrosa inflamatória (40\%). Torrão $(1999)^{(11)}$ realizou um levantamento epidemiológico de biópsias da região bucomaxilofacial e verificou que esta patologia foi a mais frequente na cavidade oral de todos 
os tipos de pacientes. Segundo Firoozmand (2005) ${ }^{(2)}$ este tipo de lesão é proveniente de uma irritação crônica de baixa intensidade. Em nossa amostra, muitos pacientes eram usuários de próteses mal-adaptadas, as quais poderiam contribuir para o desenvolvimento deste processo adaptativo. Além disso, alguns indivíduos relataram hábitos deletérios como mordedura e sucção de lábio e/ou vestíbulo e tendência a traumatismo dos tecidos moles intraorais em virtude da diminuição e/ou ausência de coordenação motora. Tais estímulos irritativos crônicos poderiam justificar a maior ocorrência da hiperplasia fibrosa inflamatória. No tocante à hiperplasia gengival, Sabbagh (2007) ${ }^{(13)}$ afirmou que em pacientes especiais que fazem uso de uso de anticonvulsivantes, estes fármacos predispõem o crescimento gengival, podendo esta hiperplasia ser agravada pelos estímulos irritativos já descritos. No presente estudo, $26,6 \%$ dos pacientes apresentaram hiperplasia de gengiva, e esta esteve associada ao uso de anticonvulsivantes.

Pacientes especiais geralmente apresentam disfunções na mastigação, deglutição e dificuldade em atingir um bom nível de higiene oral o que pode propiciar o acúmulo de biofilme. ${ }^{(14)} \mathrm{A}$ alteração da microbiota oral pode predispor o desenvolvimento de espécies oportunistas, como a Candida albicans. Em nosso estudo, a Candidíase se mostrou presente em $11,6 \%$ dos pacientes examinados e na maioria das vezes, esteve associada à diabetes mellitus. Pacientes portadores de epilepsia e outros distúrbios neurológicos também exibiram esta infecção, fato este que pode ser justificado pela alteração dos nichos ecológicos na cavidade oral em virtude da ocorrência de um quadro clínico que evidencia má higiene oral e desta forma, predispõe o desenvolvimento de espécies oportunitas a exemplo da Candida albicans.

Uma outra alteração observada foi a estomatite aftosa. Woo, em 1996 ${ }^{(15)}$ classificou a estomatite aftosa recorrente como uma doença inflamatória associada a alterações imunológicas e/ou fatores locais como trauma, estresse, ingestão de alimentos ácidos, drogas, alterações hormonais, deficiência de vitaminas e agentes infecciosos que comumen- te acometem a mucosa oral. Conforme já mencionado, os pacientes portadores de desordens neuropsicomotoras, em especial, apresentam grande dificuldade no manejo da escova e fio dental além de história de traumatismos repetitivos em determinadas áreas da mucosa oral, fato este que poderia justificar o desenvolvimentos de lesões aftosas.

Pacientes portadores de doenças cardiovasculares, pulmonares, do aparelho geniturinário, e desordens de natureza endócrina, metabólica, imunológica, hematológica, oncológica, distúrbios psiquiátricos, neurológicos e comportamentais fazem parte da classificação de pacientes especiais. (2) No presente estudo, as doenças neuropsicomotoras mais encontradas durante a realização da coleta de dados foram a paralisia cerebral, o autismo, distúrbios neurológicos, Alzheimer e paraplegia, as quais abrangeram cerca de $41 \%$ dos examinados. Entre as síndromes genéticas destacaram-se a de Sturge Weber, Down e Wilson que foram identificadas em $26,6 \%$ dos pacientes da amostra. Sabe-se que $\mathrm{o}$ atendimento odontológico a esta população representa um desafio adicional no tocante à necessidade de desenvolvimento, por parte dos profissionais de saúde, de habilidades essenciais para o manejo destes pacientes. Entre estas, podem ser incluídas a necessidade de sedação, contenção e avaliação do déficit cognitivo. ${ }^{(16)}$ Embora não tenha sido objeto de investigação desta pesquisa avaliar a formação especializada do profissional de saúde para 0 atendimento a esta população, este trabaIho permitiu ampliar o conhecimento acerca das necessidades e alterações bucais dos pacientes especiais usuários do Sistema único de Saúde (SUS). É de grande relevância proporcionar o desenvolvimento de um olhar mais cuidadoso por parte do cirurgião dentista no tocante às alterações bucais que estes indivíduos comumente apresentam.

\section{CONSIDERAÇÕES FINAIS}

Embora as lesões bucais mais prevalentes neste estudo tenham sido a hiperplasia fibrosa inflamatória, a hiperplasia gengival e a candidíase, outras 
alterações bucais foram relatadas, a exemplo de xerostomia ou sialorreia. Observou-se que as lesões orais encontradas estavam relacionadas diretamente à higiene oral deficiente e agentes traumáticos de baixa intensidade. Espera-se que os resultados do presente estudo possam contribuir para o melhor entendimento da condição de saúde bucal dos pacientes especiais, uma vez que a atenção especializada dedicada a esta população é essencial para o êxito do tratamento odontológico.

\section{REFERÊNCIAS}

1. Conselho Federal de Odontologia (Brasil). Resolução 22/2001 de 27 de dezembro de 2001. Normas sobre anúncio e exercício das especialidades odontológicas e sobre cursos de especialização. [acesso em 10 de maio de 2013]. Disponível em: http:www.cfo.org.br/

2. Little JM, Falace DA, Miller CS, Rhodus NL. Manejo Odontológico do Paciente Clinicamente Comprometido. Rio de Janeiro: Elsevier; 2008.

3. Turner RJ, Sugiya H. Understanding salivary fluid and protein secretion. Oral dis. 2002;8: 3-11.

4. Scully CCBE. Drug effects on salivary glands: dry mouth. Salivary glands and saliva. Oral dis. 2003;9:165-76.

5. Amaral SM, Miranda AMMA, Pires FR. Reações medicamentosas na cavidade oral: aspectos relevantes na Estomatologia. Rev. bras. odontol. 2009;66(1):41-53.

6. Oliveira AC, Czeresnia D, Paiva SM, Campos $M R$, Ferreira EF. Utilization of oral health care for Down syndrome patients. Rev. saúde pública. 2008;42(4):693-9.

7. Dehaitem MJ, Ridley K, Kerschbaum WE, Inglehart MR. Dental Hygiene Education About Patients with Special Needs: A Survey of U.S. Programs. J. dent. educ. 2008;72(9):10101019.
8. American Association on Intellectual and Developmental Disabilities (USA). Intellectual disability [serial online]. [acesso em maio de 2013] Disponível em: www.aamr.org/ content_104.cfm.

9. Katz CR. Integrated approach to outpatient dental treatment of a patient with cerebral palsy: a case report. Spec. care dentist. 2012;32:210-217.

10. Tomita Emy N, Fagote Ferrarezi B, Programa Educativo em Saúde Bucal para Pacientes Especiais. Odontologia e Sociedade. 1999;1(1/2):45-50

11. Torrão, A.C.R. Levantamento epidemiológico de biópsias da região bucomaxilofacial, encaminhadas ao laboratório de patologia bucal da Faculdade de Odontologia de Pernambuco. Rev. Cons. Reg. Odontol. Pernambuco. 1999;2(2):118-125.

12. Macedo Firoozmand L, Dias Almeida J, Guimaraes Cabral LA. Study of denture-induced fibrous hyperplasia cases diagnosed from 1979 to 2001. Quintessence int. 2005;36(10):825-9.

13. Sabbagh-Haddad A., Magalhães MHG. Introdução. In: Sabbagh-Haddad A. et al. Odontologia para Pacientes com Necessidades Especiais. São Paulo: Editora Santos; 2007; Cap. 1, p. 1-5.

14. Narvai PC, Antunes JLF. Saúde Bucal: a autopercepção da mutilação e das incapacidades. Projeto Sabe; 2003;1:121-4O

15. Woo SB, Sonis ST. Recurrent aphthous ulcers: a review of diagnosis and treatment. J. Am. Dent. Assoc. 1996;127;12O2-213.

16. Abreu KCS, Franco SOB, Calheiros PR. Abordagem odontológica para pacientes portadores de distúrbios neuropsicomotores. Revista Científica Eletrônica. 2009;1(1):1-20. Disponível em: http://www.facimed.edu.br/site/ revista/?onChange=Ler\&ID=21 